\title{
Fracture Toughness of Carbon Nanotube-Reinforced Metal- and Ceramic-Matrix Composites
}

\author{
Y. L. Chen, ${ }^{1,2}$ B. Liu, ${ }^{1}$ Y. Huang, ${ }^{3}$ and K. C. Hwang ${ }^{1}$ \\ ${ }^{1}$ AML, Department of Engineering Mechanics, Tsinghua University, Beijing 100084, China \\ ${ }^{2}$ Department of Mechanical Engineering, The Ohio State University, Columbus, OH 43210, USA \\ ${ }^{3}$ Department of Civil and Environmental Engineering and Department of Mechanical Engineering, Northwestern University, \\ Evanston, IL 60208, USA \\ Correspondence should be addressed to B. Liu, liubin@tsinghua.edu.cn
}

Received 15 July 2010; Accepted 1 November 2010

Academic Editor: Teng Li

Copyright () 2011 Y. L. Chen et al. This is an open access article distributed under the Creative Commons Attribution License, which permits unrestricted use, distribution, and reproduction in any medium, provided the original work is properly cited.

Hierarchical analysis of the fracture toughness enhancement of carbon nanotube- (CNT-) reinforced hard matrix composites is carried out on the basis of shear-lag theory and facture mechanics. It is found that stronger CNT/matrix interfaces cannot definitely lead to the better fracture toughness of these composites, and the optimal interfacial chemical bond density is that making the failure mode just in the transition from CNT pull-out to CNT break. For hard matrix composites, the fracture toughness of composites with weak interfaces can be improved effectively by increasing the CNT length. However, for soft matrix composite, the fracture toughness improvement due to the reinforcing CNTs quickly becomes saturated with an increase in CNT length. The proposed theoretical model is also applicable to short fiber-reinforced composites.

\section{Introduction}

Carbon nanotubes (CNTs) possess exceptionally superior physical and mechanical properties, such as high strength, low density, high flexibility, and high toughness and therefore hold great promise for employment as reinforcements in advanced composites [1-10]. However, experimental and numerical studies show that the performance of such composites depends critically on the CNT/matrix interfacial characteristics [11-13]. Interface strength and interface length are two of the most important factors that affect the mechanical properties of CNT-reinforced composites and therefore have drawn the attention of many researchers.

As a type of extraordinary reinforcements, CNTs can be incorporated in a polymer, metal, or ceramic matrix. The focus of many previous studies in CNT-reinforced composites has been on polymer-matrix materials [1421], and researchers have tried in various ways, such as nonionic surfactant and ion bombardment $[11,16,22,23]$, to form covalent bonds between CNTs and the polymer matrix to strengthen the interface. In order to know whether longer CNTs and stronger interfaces definitely result in better mechanical properties of CNT-reinforced composites, Chen et al. [24] studied the fracture toughness enhancement of CNT-reinforced polymer-matrix composites. They found that neither longer reinforcing CNTs nor stronger CNT/matrix interfaces can definitely lead to the better fracture toughness, and the optimal interfacial chemical bond density and the optimal CNT length are those making the failure mode just in the transition from CNT pull-out to CNT break.

Meanwhile, the production and application of CNTreinforced metal- and ceramic-matrix composites draw more and more attention. Ma and coworkers [25] formed CNTnano-silicon-carbide ( $\mathrm{SiC}$ )/ceramic composites and reported a $10 \%$ improvement in the strength and fracture toughness as compared to the monolithic ceramics. These modest improvements are attributed to nanotube/matrix debonding and crack deflection. The techniques to form CNT/metaloxide composites as well as $\mathrm{CNT} /$ metal-matrix composites have been developed [26-31]. However, they did not provide the expected improvement in mechanical properties. Is Chen et al.'s conclusion [24] still valid for these hard matrix composites? Is there some difference between the soft matrix 
and hard matrix CNT-reinforced composites? This paper will focus on the case with higher matrix stiffness and study the influence factors on the fracture toughness of CNTreinforced composites.

\section{Roadmap for Hierarchical Failure Analysis of CNT-Reinforced Composites}

In CNT-reinforced composites with macroscopic cracks, the high strength of CNTs can retard crack propagation, and a fracture zone bridged by CNTs at the crack tip is formed, as shown in Figure 1(a). This toughening effect of bridging CNTs is equivalent to that of nonlinear springs connecting the upper and lower crack surfaces, as shown in Figure 1(b). The force-displacement relation for these springs can be obtained by studying the pulling force $F$ and pull-out displacement $\delta$ of a single CNT, as in Figure 1(c). This $F-\delta$ curve depends on the interfacial atomic bond properties, that is, on the interaction between atoms, as shown in Figure 1(d). Therefore, CNT-reinforced composites have three failure mode levels: a bond break at the atomistic level, CNT fiber failure mode at the mesoscopic level, and macroscopic crack propagation at the macroscopic level. To better understand and optimize the toughness of CNT-reinforced composites, this paper presents hierarchical failure analysis. We first adopt shear-lag theory to investigate CNT fiber in Section 3 and then use facture mechanics to study macroscopic-level failure in Section 4. Our conclusions are summarized in the last section.

\section{Force-Displacement Relation of a Single CNT Pulled from the Matrix}

There are a large number of continuum mechanics studies on the fiber-reinforced composites, especially the widely used shear-lag theory [32-37]. Chon and Sun [33] studied stress distribution along a single reinforcing fiber of a randomly oriented chopped-fiber composite under the assumption of perfect bonding. Lawrence [34] assumed the stiffness of the fiber is lower than that of the matrix and investigated fiber pull-out from an elastic matrix. For ceramic composites, Marshall et al. [35] studied the cracking in brittle matrix. Hutchinson and Jensen [36] treated debonding process as a mode 2 crack to study fiber debonding and pull-out, and based on these studies, Budiansky et al. [37] accounted for an interfacial debonding resistance and studied the effects of debonding and initial stress on overall composite toughness. Many of these models are also applicable to CNT-reinforced composites. However, for the completeness and convenience to readers, the related analysis is still briefly presented in this section.

3.1. Shear-Lag-Model-Based Stress Analysis of the CNT and Matrix. According to the shear-lag theory, the interaction between the CNTs and the matrix that results from the chemical bonds shown in Figure 2 is shear stress, which is related to the relative displacement between the CNT fiber and the matrix $\Delta u$. A bond break occurs when $\Delta u$ reaches the critical shear displacement $\delta_{b}$, which depends only on the type of functionalization bond at the interface, whereas the corresponding interface strength $\tau_{b}$ also depends on the interface bond density. The interface shear stress $\tau$ is assumed to be proportional to the relative displacement $\Delta u$, that is,

$$
\tau(x)=k \Delta u(x)=k\left[u_{m}(x)-u_{f}(x)\right],
$$

where $k=\tau_{b} / \delta_{b}$ is the shear stiffness of the interface and $u_{m}(x)$ and $u_{f}(x)$ are the axial or $x$-direction displacements of the matrix and the CNT fiber, respectively.

Suppose the CNT and the matrix are both linear elastic, with Young's modulus $E_{f}$ and $E_{m}$, respectively. A representative volume element (RVE) including a single CNT with embedded length $L$ and diameter $d$, as shown in Figure 2, is adopted for analysis. With the balance conditions of the fiber and the matrix, the shear stress distribution can be derived [24]

$$
\begin{aligned}
\tau(x)= & F \sqrt{\frac{\tau_{b}}{C \delta_{b}}} \\
& \cdot \frac{1 /\left(E_{m} A_{m}\right) \cosh \left(x \sqrt{C \tau_{b} / \delta_{b}}\right)}{\sinh \left(L \sqrt{C \tau_{b} / \delta_{b}}\right)} \\
& +\frac{1 /\left(E_{f} A_{f}\right) \cosh \left[(x-L) \sqrt{C \tau_{b} / \delta_{b}}\right]}{\sinh \left(L \sqrt{C \tau_{b} / \delta_{b}}\right)}
\end{aligned}
$$

where $A_{f}$ and $A_{m}$ are the cross-section areas of the CNT and the matrix in the RVE, $F$ is the pulling force, and $C$ depends on the material constants and geometry parameters as

$$
C=\pi d\left(\frac{1}{E_{f} A_{f}}+\frac{1}{E_{m} A_{m}}\right) .
$$

The distribution of the axial normal stress in the CNT can also be derived as

$$
\sigma(x)=\frac{1}{A_{f}}\left[F-\int_{0}^{x} \pi d \tau(x) \mathrm{d} x\right] .
$$

3.2. Critical Pull-Out/Break Condition of CNTs. The two main fiber-level failure modes are usually interfacial debonding and fiber break, depending on the interfacial shear stress and the axial normal stress, respectively. When the composite is under increasing tension, both the shear stress on the interface and the axial normal stress in the CNT increase.

3.2.1. Critical Condition for CNT Fiber Break. Obviously, the maximum axial normal stress in the CNT is located at position $x=0$ and can be expressed as

$$
\left.\sigma(x)\right|_{\max }=\sigma(0)=\frac{F}{A_{f}} .
$$

CNT break occurs when the maximum axial normal stress reaches CNT strength $\sigma_{f}^{b}$, and the corresponding critical pulling force is

$$
F_{\max }^{\sigma}=\sigma_{f}^{b} A_{f}
$$




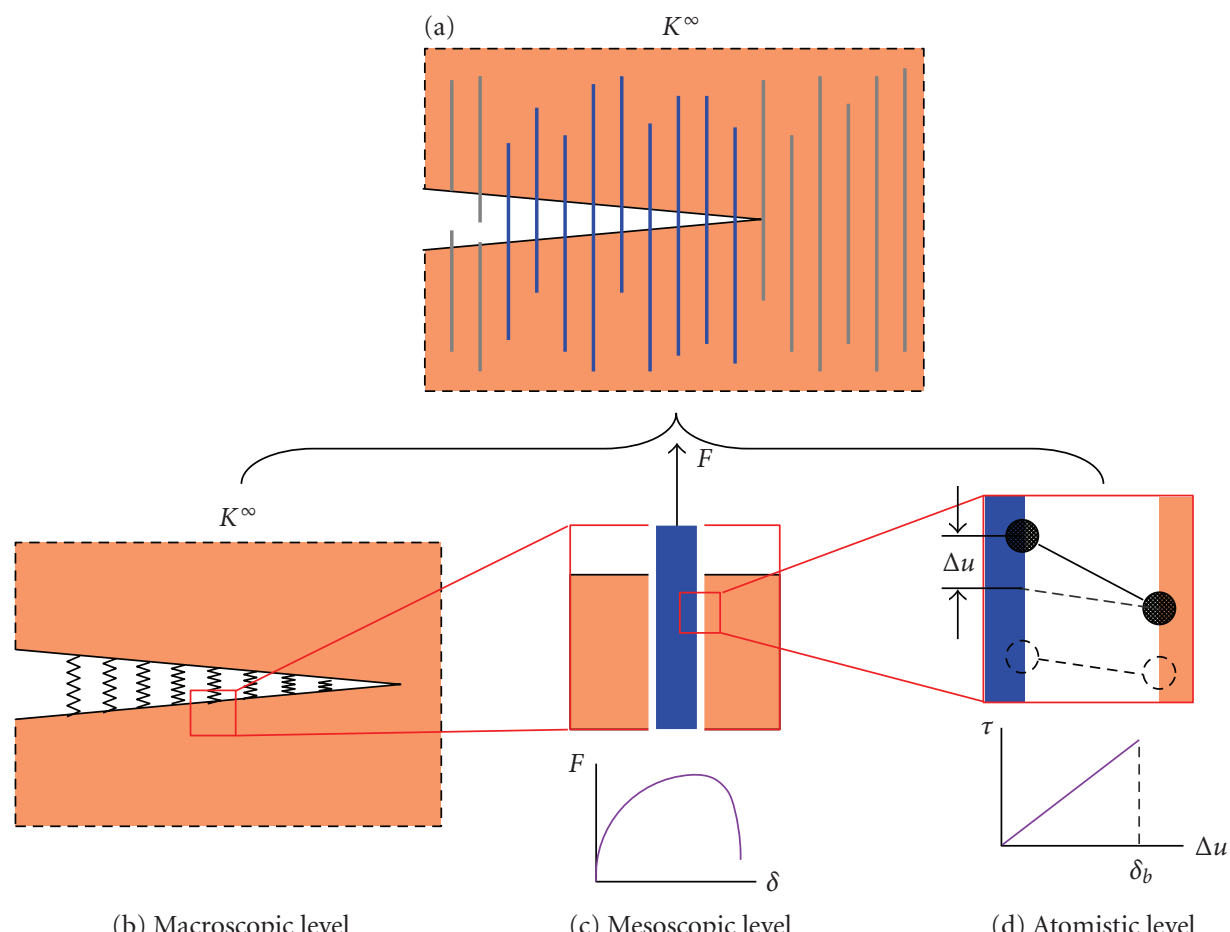

FIGURE 1: Schematic diagram of three-level failure analysis models. (a) Fracture zone bridged with CNTs at the crack tip. (b) Macroscopiclevel model with equivalent bridging nonlinear springs. (c) Mesoscopic-level model for studying CNT-fiber failure and obtaining the forcedisplacement relation of equivalent nonlinear spring. (d) Atomistic-level failure model for characterizing CNT/matrix interfacial bond breaking.

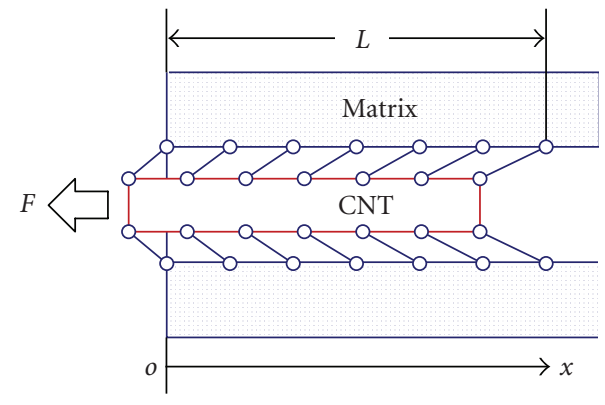

(a)

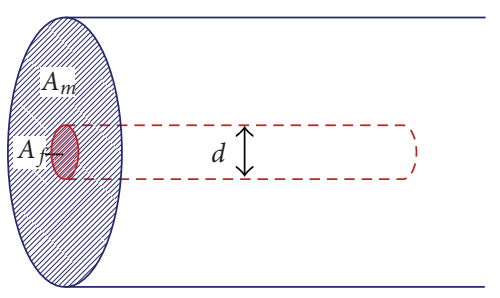

(b)

FIGURE 2: Schematic diagram of shear-lag model for the interactions between the CNT and the matrix (a), and the geometric parameters (b).

3.2.2. Critical Condition for Interfacial Debonding. According to (2), the maximum shear stress on the interface may appear at either $x=0$ for $E_{f} A_{f}<E_{m} A_{m}$ or $x=L$ for $E_{f} A_{f}>$ $E_{m} A_{m}$. In CNT/polymer composites, the stiffness of the CNT is usually greater than that of the matrix, that is, $E_{f} A_{f}>$ $E_{m} A_{m}$, as Chen et al. have previously discussed in [24], but in metal- and ceramic-matrix composites Young's modulus of the matrix is close to that of the CNTs, so the stiffness of the CNT is less than that of the matrix, that is, $E_{f} A_{f}<E_{m} A_{m}$. The current paper is focused on this hard matrix regime and sometimes also presents the results of soft matrix regime for comparison.
(1) $E_{f} A_{f}<E_{m} A_{m}$ Case (Hard Matrix). From (2), the maximum shear stress on the interface can be found at the $x=0$ position, and

$$
\begin{aligned}
\left.\tau(x)\right|_{\max }= & \tau(0) \\
= & F \sqrt{\frac{\tau_{b}}{C \delta_{b}}} \\
& \cdot \frac{1 /\left(E_{m} A_{m}\right)+1 /\left(E_{f} A_{f}\right) \cosh \left(L \sqrt{C \tau_{b} / \delta_{b}}\right)}{\sinh \left(L \sqrt{C \tau_{b} / \delta_{b}}\right)} .
\end{aligned}
$$


The corresponding critical pulling force is

$$
F_{\max }^{\tau 0}=\frac{\sqrt{C \tau_{b} \delta_{b}} \sinh \left(L \sqrt{C \tau_{b} / \delta_{b}}\right)}{1 /\left(E_{m} A_{m}\right)+1 /\left(E_{f} A_{f}\right) \cosh \left(L \sqrt{C \tau_{b} / \delta_{b}}\right)} .
$$

Together with (6) and (8), the transition condition between the pull-out and break failure modes is

$$
\begin{gathered}
\frac{\sigma_{f}^{b} A_{f}}{\sqrt{C \tau_{b} \delta_{b}}} \frac{1 /\left(E_{m} A_{m}\right)+1 /\left(E_{f} A_{f}\right) \cosh \left(L \sqrt{C \tau_{b} / \delta_{b}}\right)}{\sinh \left(L \sqrt{C \tau_{b} / \delta_{b}}\right)}>1 \\
\text { (interfacial debonding }), \\
\frac{\sigma_{f}^{b} A_{f}}{\sqrt{C \tau_{b} \delta_{b}}} \frac{1 /\left(E_{m} A_{m}\right)+1 /\left(E_{f} A_{f}\right) \cosh \left(L \sqrt{C \tau_{b} / \delta_{b}}\right)}{\sinh \left(L \sqrt{C \tau_{b} / \delta_{b}}\right)}<1
\end{gathered}
$$

(CNT fiber breaking),

that is, a weak interface results in interfacial debonding and fiber pull-out, whereas a strong interface leads to fiber break.

(2) $E_{f} A_{f}>E_{m} A_{m}$ Case (Soft Matrix). The most important difference from the hard matrix case (i.e., $E_{f} A_{f}<E_{m} A_{m}$ ) is that the maximum shear stress on the interface is found at the $x=L$ position, so the critical pulling force is [24]

$$
F_{\max }^{\tau L}=\frac{\sqrt{C \tau_{b} \delta_{b}} \sinh \left(L \sqrt{C \tau_{b} / \delta_{b}}\right)}{1 /\left(E_{m} A_{m}\right) \cosh \left(L \sqrt{C \tau_{b} / \delta_{b}}\right)+1 /\left(E_{f} A_{f}\right)},
$$

and the transition condition between the pull-out and break failure modes is [24]

$$
\begin{gathered}
\frac{\sigma_{f}^{b} A_{f}}{\sqrt{C \tau_{b} \delta_{b}}} \frac{1 /\left(E_{m} A_{m}\right) \cosh \left(L \sqrt{C \tau_{b} / \delta_{b}}\right)+1 /\left(E_{f} A_{f}\right)}{\sinh \left(L \sqrt{C \tau_{b} / \delta_{b}}\right)}>1 \\
\text { interfacial debonding, } \\
\frac{\sigma_{f}^{b} A_{f}}{\sqrt{C \tau_{b} \delta_{b}}} \frac{1 /\left(E_{m} A_{m}\right) \cosh \left(L \sqrt{C \tau_{b} / \delta_{b}}\right)+1 /\left(E_{f} A_{f}\right)}{\sinh \left(L \sqrt{C \tau_{b} / \delta_{b}}\right)}<1 \\
\text { CNT fiber breaking. }
\end{gathered}
$$

3.3. Relation between Pulling Force and Pull-Out Displacement. For different possible failure modes, there are three types of $F-\delta$ curves, as shown in Figures 3(a), 3(b), and $3(\mathrm{c})$. Here, $F$ is the pulling force, and $\delta=\Delta u(0)$ is the pullout displacement. The corresponding relations are given as below.

3.3.1. CNT Break Case. The $F-\delta$ relation can be obtained as

$$
F= \begin{cases}\delta \sqrt{\frac{C \tau_{b}}{\delta_{b}}} \cdot \frac{E_{f} A_{f} \sinh \left(L \sqrt{C \tau_{b} / \delta_{b}}\right)}{\cosh \left(L \sqrt{C \tau_{b} / \delta_{b}}\right)+1 / \alpha}, & 0 \leq \delta \leq \delta_{C}^{\sigma}, \\ 0, & \delta>\delta_{C}^{\sigma},\end{cases}
$$

where $\alpha=E_{m} A_{m} /\left(E_{f} A_{f}\right)$ is the stiffness ratio of the matrix to the CNT, and

$$
\delta_{C}^{\sigma}=\sigma_{f}^{b} \sqrt{\frac{\delta_{b}}{C \tau_{b}}} \cdot \frac{\cosh \left(L \sqrt{C \tau_{b} / \delta_{b}}\right)+1 / \alpha}{E_{f} \sinh \left(L \sqrt{C \tau_{b} / \delta_{b}}\right)} .
$$

3.3.2. Interface Debonding from the End of Pulling Force (Hard Matrix). If $E_{f} A_{f}<E_{m} A_{m}$, the interface begins to debond at the $x=0$ position where the relative displacement reaches $\delta_{b}$. So the critical pull-out displacement for the interfacial debonding case is

$$
\delta_{C}^{\tau 0}=\delta_{b} .
$$

At this time, the relative displacement at the $x=L$ position is $\delta_{b} \cdot(1 / \alpha)\left(1+\left(\alpha^{2}-1\right) /\left(\alpha \cosh \left(L \sqrt{C \tau_{b} / \delta_{b}}\right)+1\right)\right)$, which is less than $\delta_{b}$, so the interface is not debonded completely and can still stand some degree of shear stress. After this point, the $F-\delta$ curve decreases until the interface debonds completely. In the process of debonding, the $\mathrm{CNT}$ and the matrix can be analyzed as a similar system but with a shorter interface length $L_{2}\left(L_{2}<L\right)$. Both the pulling force $F$ and the pull-out displacement $\delta$ are dependent on the new interface length $L_{2}$ as follows:

$$
\begin{gathered}
F\left(L_{2}\right)=\sqrt{C \tau_{b} \delta_{b}} \cdot \frac{E_{f} A_{f} \sinh \left(L_{2} \sqrt{C \tau_{b} / \delta_{b}}\right)}{\cosh \left(L_{2} \sqrt{C \tau_{b} / \delta_{b}}\right)+1 / \alpha}, \\
\delta\left(L_{2}\right)=\delta_{b}+\left(L-L_{2}\right)\left(\sqrt{C \tau_{b} \delta_{b}} \cdot \frac{\sinh \left(L_{2} \sqrt{C \tau_{b} / \delta_{b}}\right)}{\cosh \left(L_{2} \sqrt{C \tau_{b} / \delta_{b}}\right)+1 / \alpha}\right) .
\end{gathered}
$$

Getting rid of $L_{2}$ from the above two equations, we can get the relation between the pulling force $F$ and the pull-out displacement $\delta$ after the beginning of interface debonding as

$$
\begin{aligned}
\delta= & \delta_{b}+\left\{L-\sqrt{\frac{\delta_{b}}{C \tau_{b}}} \ln \left[\frac{F+\sqrt{F^{2}+\alpha^{2}\left(C \tau_{b} \delta_{b} E_{f}^{2} A_{f}^{2}-F^{2}\right)}}{\alpha\left(E_{f} A_{f} \sqrt{C \tau_{b} \delta_{b}}-F\right)}\right]\right\} \\
& \times\left(\frac{F}{E_{f} A_{f}}\right) .
\end{aligned}
$$

So the relation between the pulling force $F$ and the relative displacement $\delta$ is

$$
F= \begin{cases}\delta \sqrt{\frac{C \tau_{b}}{\delta_{b}}} \cdot \frac{E_{f} A_{f} \sinh \left(L \sqrt{C \tau_{b} / \delta_{b}}\right)}{\cosh \left(L \sqrt{C \tau_{b} / \delta_{b}}\right)+1 / \alpha}, & 0 \leq \delta \leq \delta_{b}, \\ F_{\text {soften }}(\delta), & \delta_{b}<\delta \leq \delta_{\max },\end{cases}
$$

where $F_{\text {soften }}(\delta)$ is solved from (16) and the maximum pullout displacement $\delta_{\max }$ is determined by

$$
\frac{\partial \delta\left(L_{2}\right)}{\partial L_{2}}=0 .
$$


This process is shown in Figure 3(b), and the critical point $\left(\delta_{C}^{\tau 0}, F_{\max }^{\tau 0}\right)$ is given by (14) and (8), respectively. It is interesting to notice that after the initial debonding at $x=$ 0 , the interface first debonds gradually and steadily, with the gradual deceasing pulling force, but when the pullout displacement reaches $\delta_{\max }$, the whole bonding interface debonds simultaneously and the pulling force drops down to zero suddenly.

3.3.3. Interface Debonding from the End Away from Pulling Force (Soft Matrix). As discussed by Chen et al. in [24], if $E_{f} A_{f}>E_{m} A_{m}$, the $F-\delta$ relation is

$$
F= \begin{cases}\delta \sqrt{\frac{C \tau_{b}}{\delta_{b}}} \cdot \frac{E_{f} A_{f} \sinh \left(L \sqrt{C \tau_{b} / \delta_{b}}\right)}{\cosh \left(L \sqrt{C \tau_{b} / \delta_{b}}\right)+1 / \alpha}, & 0 \leq \delta \leq \delta_{C}^{\tau L}, \\ E_{m} A_{m} \sqrt{\frac{C \tau_{b}}{\delta_{b}}\left(\frac{\delta_{b}{ }^{2}-\delta^{2}}{1-\alpha^{2}}\right),} & \delta_{C}^{\tau L}<\delta \leq \delta_{b},\end{cases}
$$

where

$$
\delta_{C}^{\tau L}=\delta_{b} \cdot\left(\alpha+\frac{1-\alpha^{2}}{\cosh \left(L \sqrt{C \tau_{b} / \delta_{b}}\right)+\alpha}\right) .
$$

The $F-\delta$ curve is shown in Figure 3(c), and the critical point $\left(\delta_{C}^{\tau L}, F_{\max }^{\tau L}\right)$ is given by (20) and (10), respectively. Different from the other debonding case in Section 3.3.2, the interface debonds gradually and steadily, until it goes down to zero.

\section{Macroscopic-Level Fracture Failure Analysis}

4.1. Fracture Toughness Enhancement $\Delta K$. In CNT-reinforced composites, crack propagation is retarded by the pulling force of the CNTs at the crack surface, the so-called "bridge-toughening effect." The displacement of the crack surface (i.e., half of the crack opening displacement) is [38]

$$
\delta=\frac{2\left(1-v_{m}^{2}\right) K_{\mathrm{IC}}}{E_{m}} \sqrt{\frac{2 r}{\pi}}=\eta K_{\mathrm{IC}} \sqrt{r},
$$

where $r$ is the distance to the crack tip and $\eta=2 \sqrt{2}(1-$ $\left.v_{m}^{2}\right) /\left(E_{m} \sqrt{\pi}\right)$ depends only on Young's modulus $E_{m}$ and Poisson's ratio $v_{m}$. According to (12), (17), and (19), for all different types of failure modes, the pulling force $F(\delta)$ can be expressed as a function of the distance to the macroscopic crack tip $r$, that is, $F(\delta)=F(\delta(r))=F(r)$. The homogenized traction on the crack surface is then

$$
p(r)=F(r)\left(A_{f}+A_{m}\right)^{-1},
$$

and the fracture toughness enhancement $\Delta K$ can be computed as [39]

$$
\Delta K=\int_{0}^{\infty} \frac{\sqrt{2} p(r)}{\sqrt{\pi r}} \mathrm{~d} r
$$

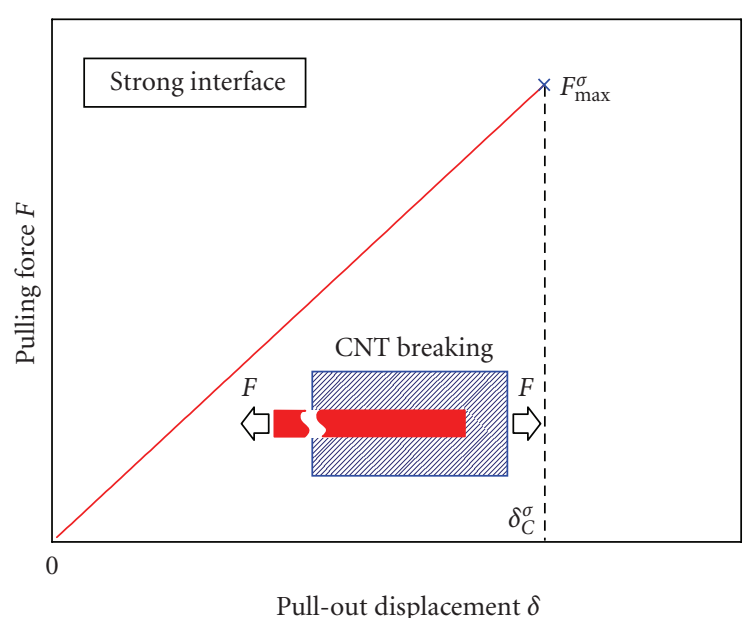

(a)

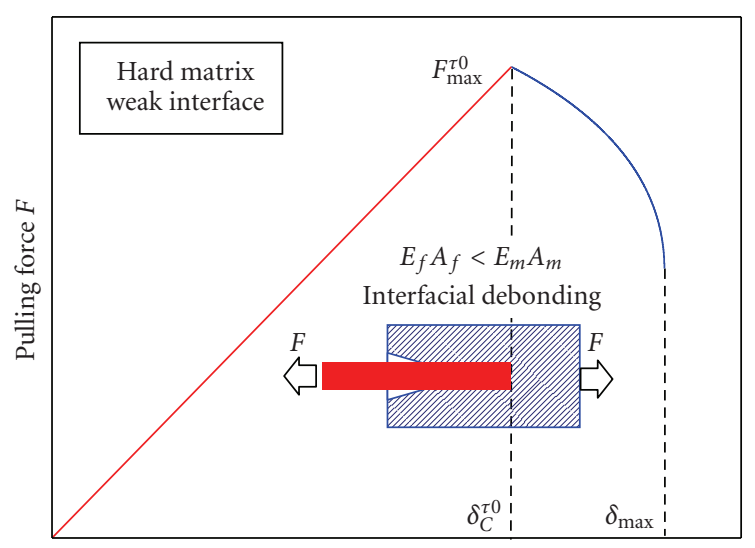

Pull-out displacement $\delta$

(b)

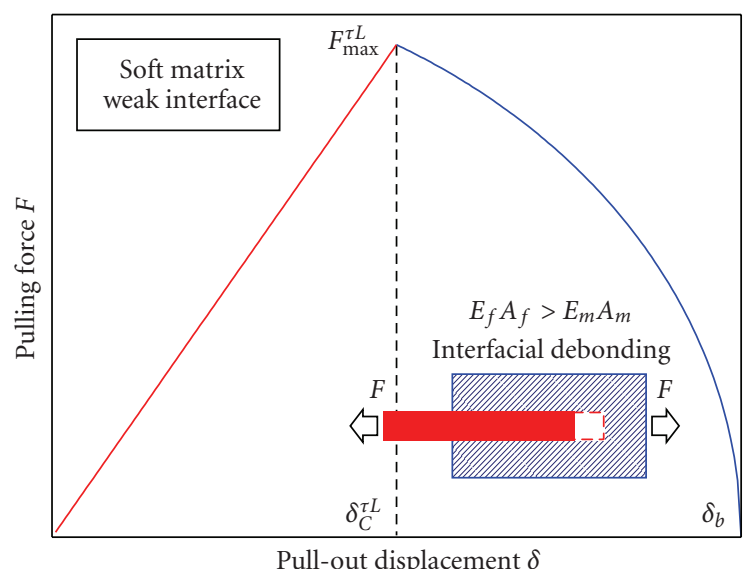

(c)

FIGURE 3: The relation between the pulling force and the pullout displacement for the three CNT-fiber failure modes (a) CNT breaking, (b) interface failure with partial steady debonding when $E_{f} A_{f}<E_{m} A_{m}$, and (c) interface failure with steady debonding when $E_{f} A_{f}>E_{m} A_{m}$. 


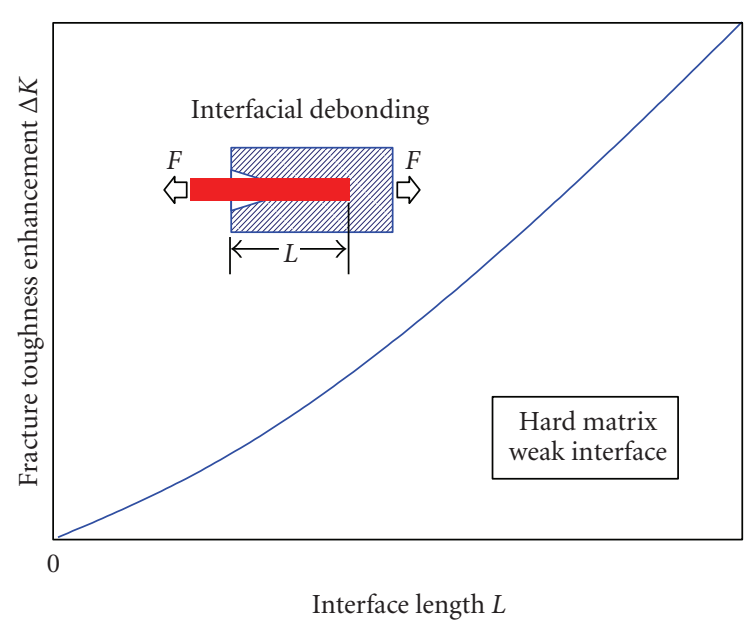

(a)

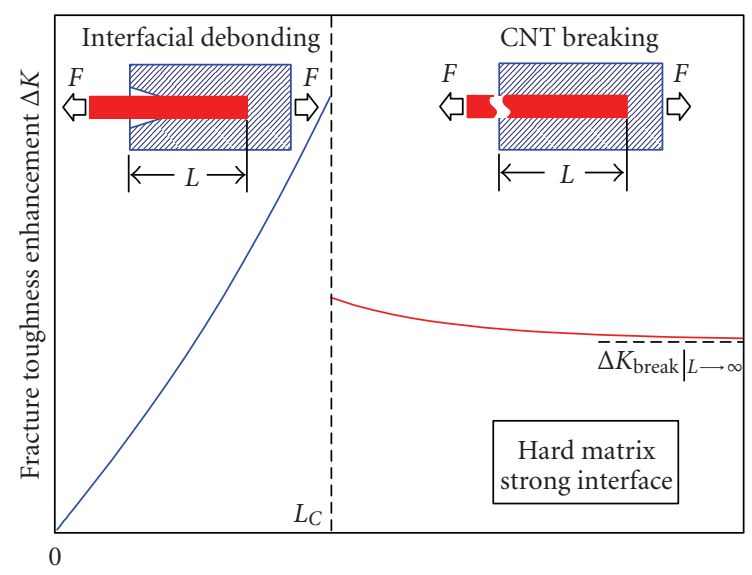

Interface length $L$

(c)

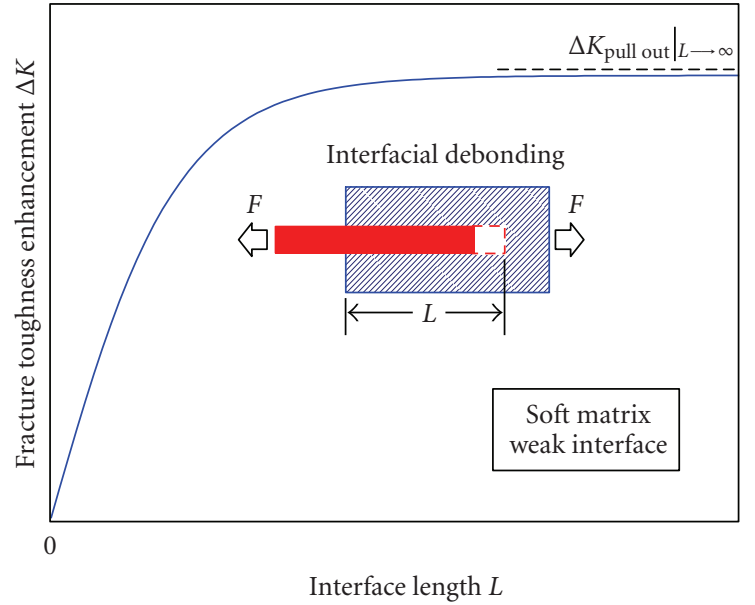

(b)

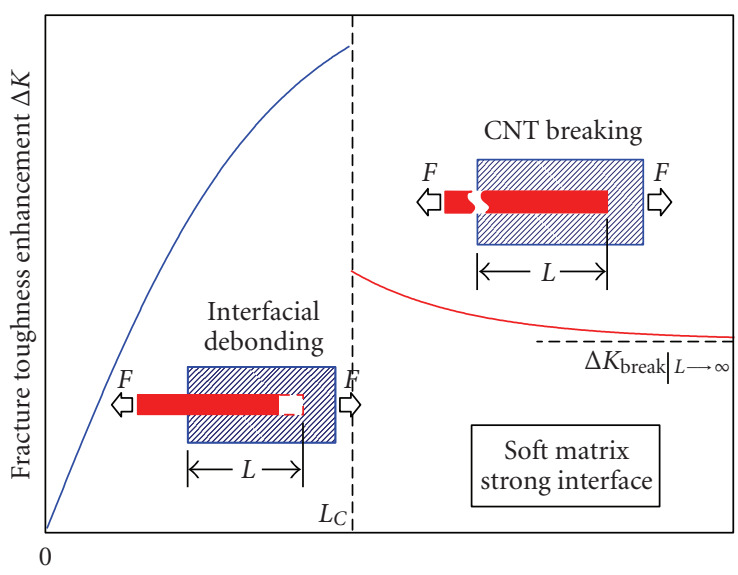

Interface length $L$

(d)

FIGURE 4: Schematic diagram for the effect of the interface length on the fracture toughness enhancement: (a) hard matrix, weak interface $\tau_{b}<\left(\sigma_{f}^{b}\right)^{2} /\left(C E_{f}^{2} \delta_{b}\right)$. (b) soft matrix, weak interface $\tau_{b}<\left(C \sigma_{f}^{b} A_{f}\right)^{2} /\left(E_{m}^{2} A_{m}^{2} \delta_{b}\right)$. (c) hard matrix, strong interface $\tau_{b}>\left(\sigma_{f}^{b}\right)^{2} /\left(C E_{f}^{2} \delta_{b}\right)$; $(\mathrm{d})$ soft matrix, strong interface $\tau_{b}>\left(C \sigma_{f}^{b} A_{f}\right)^{2} /\left(E_{m}^{2} A_{m}^{2} \delta_{b}\right)$.

4.2. Factors Affecting the Fracture Toughness Enhancement $\Delta K$. For the case of hard matrix $\left(E_{f} A_{f}<E_{m} A_{m}\right)$, the factors affecting the fracture toughness enhancement $\Delta K$ are discussed below, and some results of soft matrix regime in [24] are also presented briefly for comparison.

\subsubsection{Effect of Interface Length L. From the pull-out/break} critical condition (9), we know that if $\tau_{b}<\left(\sigma_{f}^{b}\right)^{2} /\left(C E_{f}^{2} \delta_{b}\right)$, the failure mode should always be the CNT pull-out, as shown in Figure 4(a). In this case, the fracture toughness enhancement $\Delta K$ increases with an increase in the interface length $L$ infinitely. This phenomenon is different from the case of weak interface in the soft matrix CNT-reinforced composites shown in Figure 4(b), in which an upper limit of the fracture toughness enhancement exists so any further lengthening of the CNTs improves the fracture toughness of the composites only slightly [24].
For the case with strong interface $\tau_{b}>\left(\sigma_{f}^{b}\right)^{2} /\left(C E_{f}^{2} \delta_{b}\right)$, as shown in Figure 4(c), a critical length $L_{C}$ exists and can be determined from the pull-out/break critical condition (9):

$$
L_{C}=\sqrt{\frac{\delta_{b}}{C \tau_{b}}} \ln \frac{\sigma_{f}^{b}+\sqrt{C \tau_{b} \delta_{b}\left(\alpha E_{f}\right)^{2}-\left(\sigma_{f}^{b}\right)^{2}\left(\alpha^{2}-1\right)}}{\alpha\left(E_{f} \sqrt{C \tau_{b} \delta_{b}}-\sigma_{f}^{b}\right)} .
$$

When $L<L_{C}$, the CNT is pulled out, and the fracture toughness enhancement $\Delta K$ increases with an increase in the interface length $L$. If $L$ is further increased beyond the critical length $L_{C}$, the failure mode is converted from CNT pull-out to $\mathrm{CNT}$ break and $\Delta K$ drops significantly. In this regime, the fracture toughness enhancement $\Delta K$ decreases with an increase in $L$ and finally approaches the following value:

$$
\left.\Delta K_{\text {break }}\right|_{L \rightarrow \infty}=\sqrt{\frac{2 \delta_{b}}{\pi C \tau_{b}}} \frac{\left(\sigma_{f}^{b}\right)^{2} A_{f}}{\left(A_{f}+A_{m}\right) \eta K_{\mathrm{IC}} E_{f}} .
$$




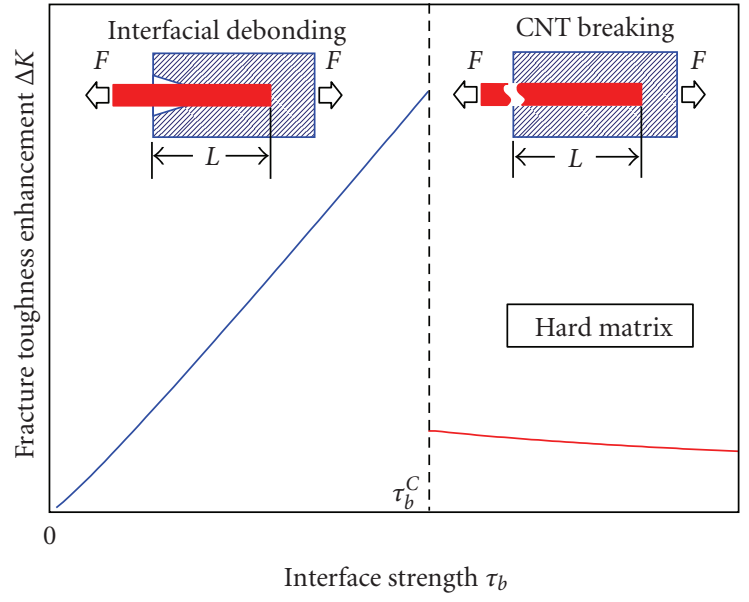

(a)

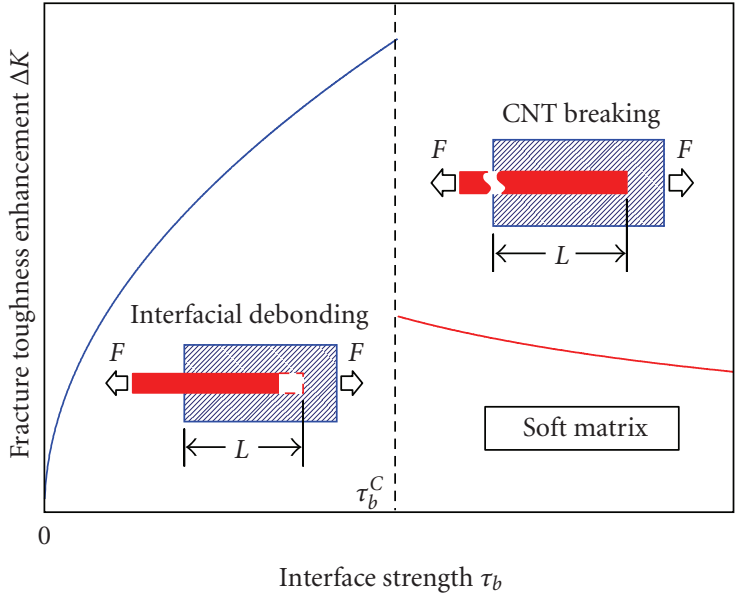

(b)

FIGURE 5: Schematic diagram for the effect of the interface strength on the fracture toughness enhancement: (a) hard matrix; (b) soft matrix.

It is interesting to note that, in this case, lengthening the interface beyond the critical length can even decrease the fracture toughness, which is very similar to the result shown is Figure 4(d) for the soft matrix CNT-reinforced composites [24].

4.2.2. Effect of Interface Strength $\tau_{b}$. Another important factor affecting the toughness enhancement is interface strength $\tau_{b}$, and its effect is shown in Figure 5(a). When $\tau_{b}$ is small, the failure mode is CNT pull-out, and the fracture toughness enhancement $\Delta K$ increases with an increase in $\tau_{b}$. Further increasing $\tau_{b}$ beyond the critical interface strength $\tau_{b}^{c}$, which can be determined by the pull-out/break critical condition (9), leads the failure mode to be converted from CNT pull-out to CNT break, and $\Delta K$ drops significantly. In this regime, the toughness enhancement $\Delta K$ decreases with an increase in $\tau_{b}$. This result agrees with the experimental and numerical studies of Xia et al's work on ceramic-matrix composites $[40,41]$, as well as the theoretical studies for soft matrix CNT-reinforced composites in Figure 5(b) [24]. Therefore, for CNTs with a given length, the maximum fracture toughness of the composite is achieved when $\tau_{b}$ is only slightly smaller than the critical interface strength $\tau_{b}^{c}$.

4.3. Optimization of Fracture Toughness Enhancement. In this subsection, we attempt to optimize the composite fracture toughness for the case of $E_{f} A_{f}<E_{m} A_{m}$ by tailoring both the interface length $L$ and interface strength $\tau_{b}$, which is essentially a bivariate optimization problem. For convenience, we use another group of variables, normalized interface length $\widehat{L}=L \sqrt{C \tau_{b} / \delta_{b}}$ and $\tau_{b}$, instead. According to (9), the maximum $\Delta K$ with a given $\hat{L}$ can be achieved when

$$
\tau_{b}=\tau_{b}^{\text {optimal }}(\hat{L})=\left(\frac{\sigma_{f}^{b} A_{f}}{E_{m} A_{m}} \frac{1+\alpha \cosh (\widehat{L})}{\sinh (\hat{L})}\right)^{2} /\left(C \delta_{b}\right) .
$$

Figure 6 shows the normalized fracture toughness enhancement $\Delta \hat{K}=\Delta K \cdot\left(A_{f}+A_{m}\right) \eta K_{\mathrm{IC}} / \sigma_{f}^{b} A_{f} \delta_{b}$ versus $\hat{L}$ for

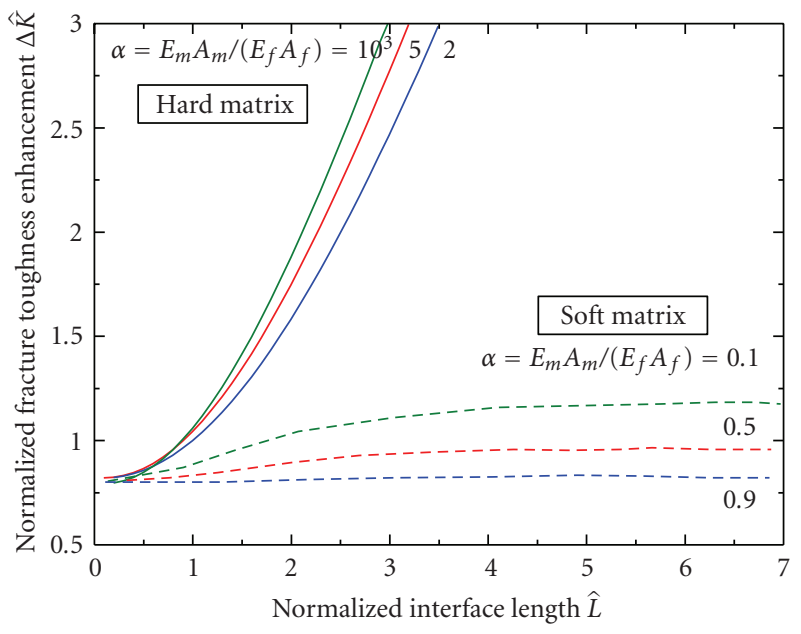

FIGURE 6: The normalized fracture toughness enhancement $\Delta \hat{K}$ as a function of the normalized interface length $\hat{L}$ with different stiffness ratio $\alpha=E_{m} A_{m} /\left(E_{f} A_{f}\right)$ and optimal interface strength.

cases with different stiffness ratios: $\alpha=E_{m} A_{m} /\left(E_{f} A_{f}\right)$. It is found that when $\widehat{L} \rightarrow \infty, \Delta K$ reaches an infinite value. And according to (26), the corresponding optimal interface strength is

$$
\left.\tau_{b}^{\text {optimal }}\right|_{\hat{L} \rightarrow \infty}=\frac{\alpha\left(\sigma_{f}^{b}\right)^{2} A_{f}}{\pi d \delta_{b} E_{f}(\alpha+1)} .
$$

This result is different from the case of $E_{f} A_{f}>E_{m} A_{m}$ studied by Chen et al. [24], which presents the upper limit of the fracture toughness enhancement, as shown in Figure 6 with the dashed lines. When $E_{f} A_{f}<E_{m} A_{m}$, the value of the dimensionless fracture toughness enhancement $\Delta \widehat{K}$ increases with $\widehat{L}$ unlimitedly, as shown in Figure 6 by the solid lines. So, there is neither upper limit for $\Delta \widehat{K}$ nor optimal value for the CNT length $L$. The optimal CNT length $L$ can only depend 
on the preparation level and some other factors such as the critical CNT length for clustering and self-folding [42].

\section{Conclusions}

Combining the shear-lag model and fracture mechanics, we have carried out the hierarchical failure analysis on CNT-reinforced composites with hard matrix. The following conclusions have been reached.

(1) Stronger CNT/matrix interfaces cannot definitely lead to a better fracture toughness of these composites. In contrast, the optimal interfacial chemical bond density is that making the failure mode just in the transition from CNT pull-out to CNT break.

(2) For composites with hard matrix, there exists a critical interface strength, below which the CNT is always pulled out, and the fracture toughness can be effectively improved by increasing the interface length $L$. However, for soft matrix composite, the fracture toughness improvement due to the reinforcing CNTs quickly becomes saturated with an increase in CNT length.

It should be noted that the theoretical analysis and conclusions drawn in this paper can also be extended to fiberreinforced composites.

\section{Acknowledgments}

The authors acknowledge the support of the National Natural Science Foundation of China (Grant nos. 10702034, 10732050, 90816006 and 10820101048) and the National Basic Research Program of China (973 Program), Grant nos. 2007 CB936803 and 2010CB832701.

\section{References}

[1] M. M. J. Treacy, T. W. Ebbesen, and J. M. Gibson, "Exceptionally high Young's modulus observed for individual carbon nanotubes," Nature, vol. 381, no. 6584, pp. 678-680, 1996.

[2] E. W. Wong, P. E. Sheehan, and C. M. Lieber, "Nanobeam mechanics: elasticity, strength, and toughness of nanorods and nanotubes," Science, vol. 277, no. 5334, pp. 1971-1975, 1997.

[3] M.-F. Yu, O. Lourie, M. J. Dyer, K. Moloni, T. F. Kelly, and R. S. Ruoff, "Strength and breaking mechanism of multiwalled carbon nanotubes under tensile load," Science, vol. 287, no. 5453, pp. 637-640, 2000.

[4] B. Vigolo, A. Penicaud, C. Coulon et al., "Macroscopic fibers and ribbons of oriented carbon nanotubes," Science, vol. 290, no. 5495, pp. 1331-1334, 2000.

[5] B. I. Yakobson, M. P. Campbell, C. J. Brabec, and J. Bernholc, "High strain rate fracture and C-chain unraveling in carbon nanotubes," Computational Materials Science, vol. 8, no. 4, pp. 341-348, 1997.

[6] G. Gao, T. Çaǧin, and W. A. Goddard III, "Energetics, structure, mechanical and vibrational properties of singlewalled carbon nanotubes," Nanotechnology, vol. 9, no. 3, pp. 184-191, 1998.

[7] Y. L. Chen, B. Liu, J. Wu, Y. Huang, H. Jiang, and K. C. Hwang, "Mechanics of hydrogen storage in carbon nanotubes," Journal of the Mechanics and Physics of Solids, vol. 56, no. 11, pp. 32243241, 2008.
[8] Y. Yin, Y. L. Chen, J. Yin, and K. Huang, "Geometric conservation laws for Y-branched carbon nanotubes," Nanotechnology, vol. 17, pp. 1-5, 2006.

[9] Y.-L. Chen, B. Liu, Y.-J. Yin, Y.-G. Huang, and K.-C. Hwuang, "Nonlinear deformation processes and damage modes of super carbon nanotubes with armchair-armchair topology," Chinese Physics Letters, vol. 25, no. 7, pp. 2577-2580, 2008.

[10] Y. Chen, Y. Yin, Y. Huang, and K.-C. Hwang, "Atomistic simulations of the nonlinear deformation and damage modes of super carbon nanotubes," Journal of Computational and Theoretical Nanoscience, vol. 6, no. 1, pp. 41-45, 2009.

[11] F. H. Gojny, J. Nastalczyk, Z. Roslaniec, and K. Schulte, "Surface modified multi-walled carbon nanotubes in CNT/epoxycomposites," Chemical Physics Letters, vol. 370, no. 5-6, pp. 820-824, 2003.

[12] S. J. V. Frankland, A. Caglar, D. W. Brenner, and M. Griebel, "Molecular simulation of the influence of chemical cross-links on the shear strength of carbon nanotube-polymer interfaces," Journal of Physical Chemistry B, vol. 106, no. 12, pp. 30463048, 2002.

[13] S. J. V. Frankland and V. M. Harik, "Analysis of carbon nanotube pull-out from a polymer matrix," Surface Science, vol. 525, no. 1-3, pp. L103-L108, 2003.

[14] P. M. Ajayan, O. Stephan, C. Colliex, and D. Trauth, "Aligned carbon nanotube arrays formed by cutting a polymer resinnanotube composite," Science, vol. 265, no. 5176, pp. 12121214, 1994.

[15] M. S. P. Shaffer and A. H. Windle, "Fabrication and characterization of carbon nanotube/poly(vinyl alcohol) composites," Advanced Materials, vol. 11, no. 11, pp. 937-941, 1999.

[16] X. Gong, J. Liu, S. Baskaran, R. D. Voise, and J. S. Young, "Surfactant-assisted processing of carbon nanotube/polymer composites," Chemistry of Materials, vol. 12, no. 4, pp. 10491052, 2000.

[17] S. Kumar, H. Doshi, M. Srinivasarao, J. O. Park, and D. A. Schiraldi, "Fibers from polypropylene/nano carbon fiber composites," Polymer, vol. 43, no. 5, pp. 1701-1703, 2002.

[18] X. Tong, X.-J. He, and H.-M. Cheng, "Effect of carbon nanotubes on mechanical properties and crystallization process of high density polyethylene," New Carbon Materials, vol. 19, no. 4, pp. 261-267, 2004.

[19] D. Qian, E. C. Dickey, R. Andrews, and T. Rantell, "Load transfer and deformation mechanisms in carbon nanotubepolystyrene composites," Applied Physics Letters, vol. 76, no. 20, pp. 2868-2870, 2000.

[20] C. Bower, R. Rosen, L. Jin, J. Han, and O. Zhou, "Deformation of carbon nanotubes in nanotube-polymer composites," Applied Physics Letters, vol. 74, no. 22, pp. 3317-3319, 1999.

[21] P. M. Ajayan, L. S. Schadler, C. Giannaris, and A. Rubio, "Single-walled carbon nanotube-polymer composites: strength and weakness," Advanced Materials, vol. 12, no. 10, pp. 750-753, 2000.

[22] B. Ni and S. B. Sinnott, "Tribological properties of carbon nanotube bundles predicted from atomistic simulations," Surface Science, vol. 487, no. 1-3, pp. 87-96, 2001.

[23] Y. Hu, I. Jang, and S. B. Sinnott, "Modification of carbon nanotube-polystyrene matrix composites through polyatomic-ion beam deposition: predictions from molecular dynamics simulations," Composites Science and Technology, vol. 63, no. 11, pp. 1663-1669, 2003.

[24] Y. L. Chen, B. Liu, X. Q. He, Y. Huang, and K. C. Hwang, "Failure analysis and the optimal toughness design of carbon nanotube-reinforced composites," Composites Science and Technology, vol. 70, no. 9, pp. 1360-1367, 2010. 
[25] R. Z. Ma, J. Wu, B. Q. Wei, J. Liang, and D. H. Wu, "Processing and properties of carbon nanotubes-nano-SiC ceramic," Journal of Materials Science, vol. 33, no. 21, pp. 52435246, 1998.

[26] E. Flahaut, A. Peigney, CH. Laurent, CH. Marlière, F. Chastel, and A. Rousset, "Carbon nanotube-metal-oxide nanocomposites: microstructure, electrical conductivity and mechanical properties," Acta Materialia, vol. 48, no. 14, pp. 3803-3812, 2000.

[27] A. Peigney, C. Laurent, O. Dumortier, and A. Rousset, "Carbon nanotubes-Fe-alumina nanocomposites. Part I: influence of the Fe content on the synthesis of powders," Journal of the European Ceramic Society, vol. 18, no. 14, pp. 1995-1104, 1998.

[28] C. Laurent, A. Peigney, O. Dumortier, and A. Rousset, "Carbon nanotubes-Fe-Alumina nanocomposites. Part II: microstructure and mechanical properties of the hot-Pressed composites," Journal of the European Ceramic Society, vol. 18, no. 14, pp. 2005-2013, 1998.

[29] A. Peigney, CH. Laurent, and A. Rousset, "Synthesis and characterization of alumina matrix nanocomposites containing carbon nanotubes," Key Engineering Materials, no. 136, pp. 743-746, 1997.

[30] X. Chen, J. Xia, J. Peng, W. Li, and S. Xie, "Carbon-nanotube metal-matrix composites prepared by electroless plating," Composites Science and Technology, vol. 60, no. 2, pp. 301-306, 2000.

[31] C. L. Xu, B. Q. Wei, R. Z. Ma, J. Liang, X. K. Ma, and D. H. $\mathrm{Wu}$, "Fabrication of aluminum-carbon nanotube composites and their electrical properties," Carbon, vol. 37, no. 5, pp. 855$858,1999$.

[32] H. L. Cox, "The elasticity and strength of paper and other fibrous materials," British Journal of Applied Physics, vol. 3, no. 1, pp. 72-79, 1952.

[33] C. T. Chon and C. T. Sun, "Stress distributions along a short fibre in fibre reinforced plastics," Journal of Materials Science, vol. 15, no. 4, pp. 931-938, 1980.

[34] P. Lawrence, "Some theoretical considerations of fibre pull-out from an elastic matrix," Journal of Materials Science, vol. 7, no. 1, pp. 1-6, 1972.

[35] D. B. Marshall, B. N. Cox, and A. G. Evans, "The mechanics of matrix cracking in brittle-matrix fiber composites," Acta Metallurgica, vol. 33, no. 11, pp. 2013-2021, 1985.

[36] J. W. Hutchinson and H. M. Jensen, "Models of fiber debonding and pullout in brittle composites with friction," Mechanics of Materials, vol. 9, no. 2, pp. 139-163, 1990.

[37] B. Budiansky, A. G. Evans, and J. W. Hutchinson, "Fibermatrix debonding effects oncracking in aligned fiber ceramic composites," International Journal of Solids and Structures, vol. 32, no. 3-4, pp. 315-328, 1995.

[38] T. L. Anderson, Fracture Mechanics: Fundamentals and Applications, CRC Press LLC, Boca Raton, Fla, USA, 1995.

[39] H. Tada, P. C. Paris, and G R. Irwin, The Stress Analysis of Cracks Handbook, ASME Press, New York, NY, USA, 3rd edition, 2000.

[40] Z. Xia, L. Riester, W. A. Curtin et al., "Direct observation of toughening mechanisms in carbon nanotube ceramic matrix composites," Acta Materialia, vol. 52, no. 4, pp. 931-944, 2004.

[41] Z. Xia and W. A. Curtin, "Tough-to-brittle transitions in ceramic-matrix composites with increasing interfacial shear stress," Acta Materialia, vol. 48, no. 20, pp. 4879-4892, 2000.

[42] W. Zhou, Y. Huang, B. Liu et al., "Self-folding of single- and multiwall carbon nanotubes," Applied Physics Letters, vol. 90, no. 7, Article ID 73107, 2007. 

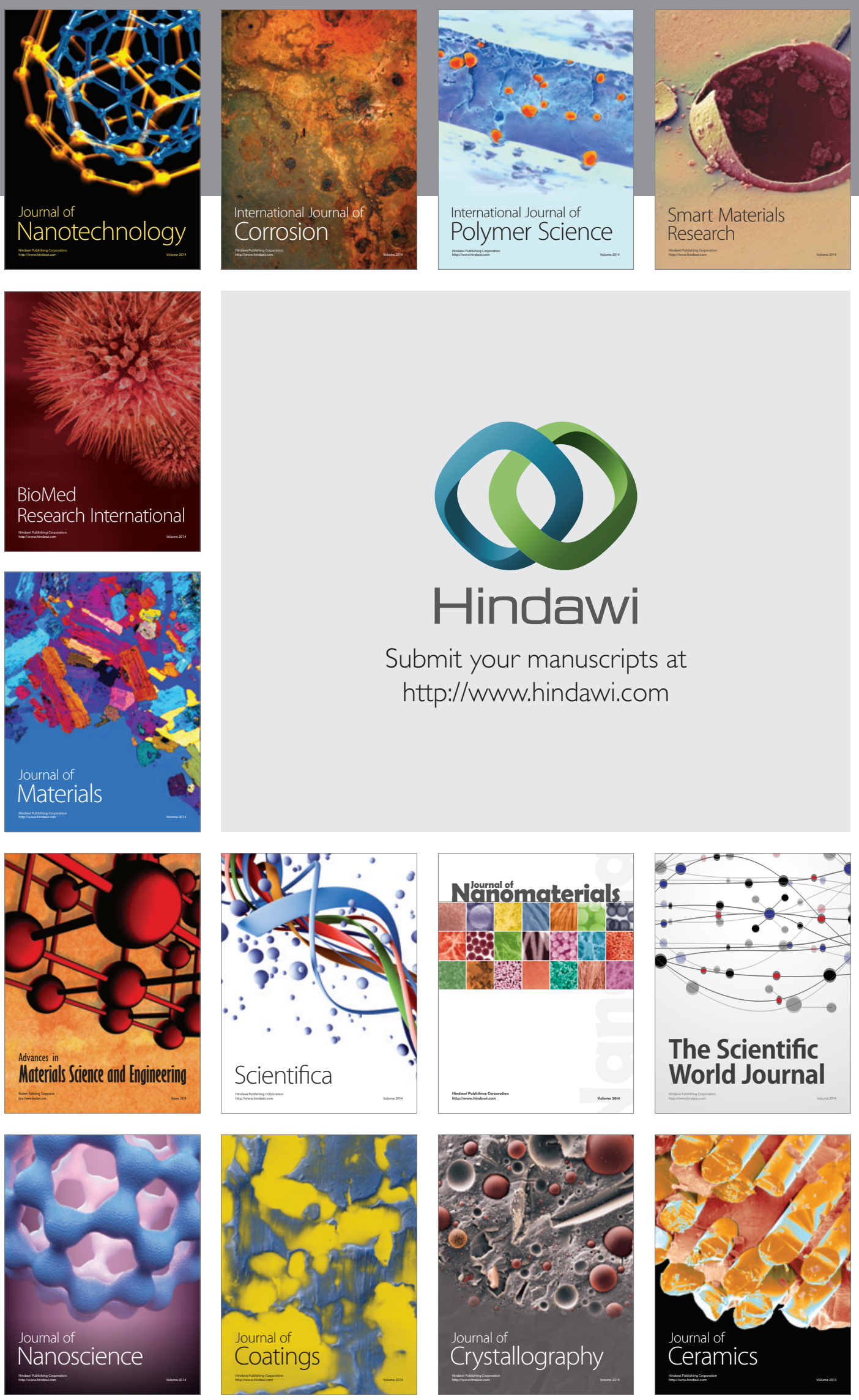

The Scientific World Journal

Submit your manuscripts at

http://www.hindawi.com

\section{World Journal}

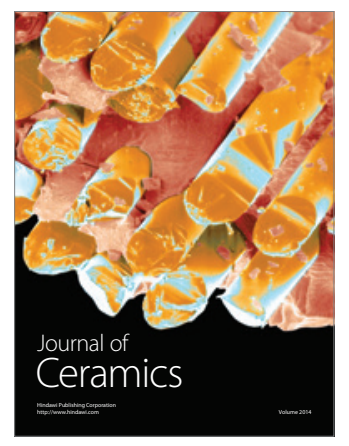

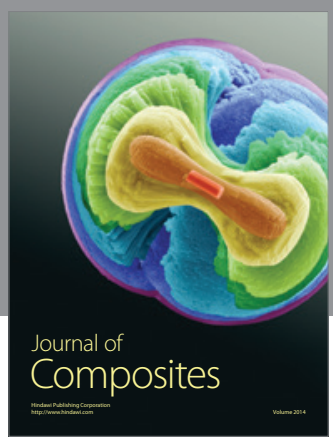
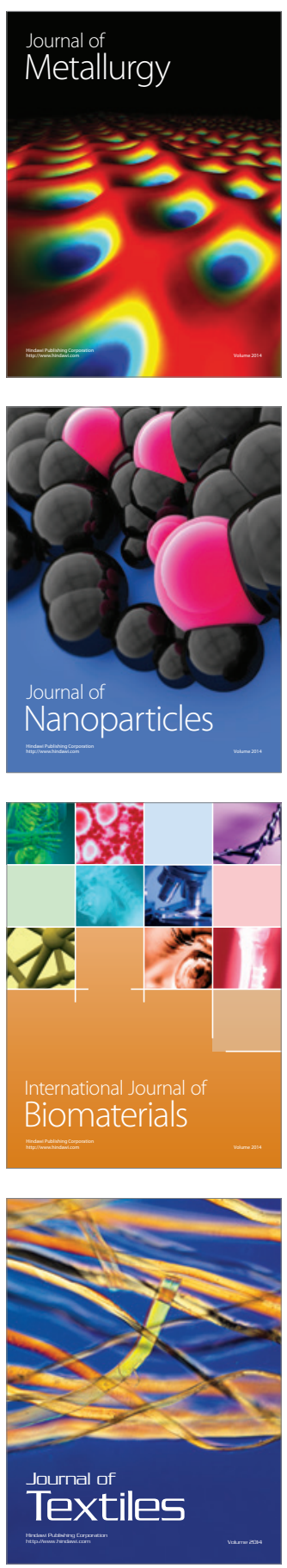